\title{
Regulator Network Analysis of Rice and Maize Yield-Related Genes
}

\author{
Zheng Chen 1,2, Zijie Shen'2, Lei Xü ${ }^{3}$, Da Zhao ${ }^{1,2}$ and Quan Zou ${ }^{2 *}$ \\ ${ }^{1}$ School of Applied Chemistry and Biological Technology, Shenzhen Polytechnic, Shenzhen, China, ${ }^{2}$ Institute \\ of Fundamental and Frontier Sciences, University of Electronic Science and Technology of China, Chengdu, China, ${ }^{3}$ School \\ of Electronic and Communication Engineering, Shenzhen Polytechnic, Shenzhen, China
}

OPEN ACCESS

Edited by:

Tao Huang,

Shanghai Institutes for Biological

Sciences, Chinese Academy of Sciences (CAS), China

Reviewed by: Renchu Guan, Jilin University, China Yushan Qiu,

Shenzhen University, China

${ }^{*}$ Correspondence:

Quan Zou

zouquan@nclab.net

Specialty section:

This article was submitted to Epigenomics and Epigenetics, a section of the journal Frontiers in Cell and Developmental Biology

Received: 26 October 2020 Accepted: 12 November 2020 Published: 03 December 2020

Citation:

Chen Z, Shen Z, Xu L, Zhao D and Zou Q (2020) Regulator Network Analysis of Rice and Maize Yield-Related Genes. Front. Cell Dev. Biol. 8:621464. doi: 10.3389/fcell.2020.621464
Rice and maize are the principal food crop species worldwide. The mechanism of gene regulation for the yield of rice and maize is still the research focus at present. Seed size, weight and shape are important traits of crop yield in rice and maize. Most members of three gene families, APETALA2/ethylene response factor, auxin response factors and MADS, were identified to be involved in yield traits in rice and maize. Analysis of molecular regulation mechanisms related to yield traits provides theoretical support for the improvement of crop yield. Genetic regulatory network analysis can provide new insights into gene families with the improvement of sequencing technology. Here, we analyzed the evolutionary relationships and the genetic regulatory network for the gene family members to predicted genes that may be involved in yield-related traits in rice and maize. The results may provide some theoretical and application guidelines for future investigations of molecular biology, which may be helpful for developing new rice and maize varieties with high yield traits.

Keywords: crop yield, gene function, genetic network, phylogenetic, rice, maize

\section{INTRODUCTION}

It has been predicted that crop yields must double to meet the demands of the rising world population by 205 (Ray et al., 2013). However, it is difficult to increase the effective cultivated area, and increasing crop yield is the only way to ensure food supply. Rice and wheat are key food crops and have been the most widely consumed staple foods in most parts of the world. They are grown as annual grain and belong to the monocotyledonous grass family. At the same time, rice and maize are model crops studied in the fields of genetics and genomics of grasses. Grain yield is a complex trait multiplicatively determined by several component traits. The number of grains per panicle, panicle number per hull, and grain weight are common traits of rice and maize (Wang and Li, 2011; Bommert et al., 2013; Yang et al., 2018; Harrop et al., 2019). ZmGS5 as the vital gene to increase the grain weight and cell number in the transgenic plants of Arabidopsis thaliana L., suggesting that ZmGS5 may have a conserved function among different plant species that affects seed development (Liu et al., 2015a). It is shown that yield-related QTLs are conserved between maize and rice (Liu et al., 2017). Another yield-related QTL is kernel row number QTL, KRN4, which can enhance grain productivity by increasing KRN per ear (Liu et al., 2015c). The increasing of endosperm also plays a role in the crop yield, therefore, $Z m G E 2$ gene has effect on the maize yield, which is associated with increase in embryo to endosperm ratio in maize (Zhang et al., 2012). The analysis of key genes for crop yield traits and their genetic regulatory networks is a scientific problem that must be solved for the improvement of crop yields. APETALA2/ethylene response factor (AP2/ERF), auxin response factors (ARFs) and MADS genes are key factors in grain yield traits and crop domestication (Harrop et al., 2019; Li et al., 2019; Wang et al., 2019). 
The AP2/ERF superfamily contains key regulators in various pathways for development and yield in plants (Riechmann and Meyerowitz, 1998). For example, ZmRAP2.7 and ZmEREB94, AP2/ERF members, participated in root development and starch synthesis, respectively (Li et al., 2017, 2019). The function of $i d s 1 / T s 6$ is the regulation of spikelet pair meristem development (Wang et al., 2019). Several AP2-like genes are key factors with respect to inflorescence branching and architecture in domesticated rice (Harrop et al., 2019). OsGL6 is involved in trichome formation in rice (Xie et al., 2020). ARFs can bind to auxin response DNA elements (AuxRE) of the genes to regulate plant development and growth (Li et al., 2016). Genes with MADS-box, a conserved sequence motif, can encode the transcription factors regulating various processes such as seed and flower development and organ differentiation in plants (Schwarz-Sommer et al., 1990; Becker and Theissen, 2003; Gramzow et al., 2010; Alvarez-Buylla et al., 2019). The ZmMADS69 allele controls maize flowering time (Liang et al., 2019). Overexpression of $z m m 28$ is associated with a significant increase in grain yield in maize (Wu et al., 2019). MADS78 and MADS79 are key regulators in rice early seed development (Paul et al., 2020). The functions of OsMADS57 are related to plant vegetative growth in rice (Chu et al., 2019).

Until now, related research on crop yield traits focused on single gene and their upstream and downstream regulatory pathways. However, there are rarely studies pertaining to genetic control networks of multiple genes. A genetic regulatory network (GRN) is a collection of molecular regulators and is composed of nodes and edges. The nodes, namely, regulators, can be DNA, RNA, proteins or complexes of these. The edges are the functional interaction model, called regulatory relationships, which can be positive activation and negative inhibition. Regulators and their functional interactions form the backbone of the cellular machinery. The network is a mechanism for controlling morphogenesis and individual development. The characteristics of genetic control of crop yield for rice and maize development are helpful for applying the traits in crop breeding (Yan and Tan, 2019). This can promote research on the genetic basis of the formation of major crop traits, and even the theoretical basis for the in-depth understanding of the common transformation mechanism of the genetic structure of the complex traits of grasses.

As shown in Figure 1, we evaluated the evolutionary relationships of three gene families, AP2, ARF, and MADS, in rice and maize, respectively. We constructed the GRN of the gene families to predict yield-related uncharacterized genes, which can provide some theoretical guidelines for future molecular biology investigations involving high yield traits in rice and maize.

\section{MATERIALS AND METHODS}

\section{DATA}

\section{RiceData}

The information of gene families related to rice is derived from RiceData ${ }^{1}$. This database can be used to query resources, such as excellent rice germplasms, rice mutants, molecular markers, genes, and pedigrees. Relevant literature searches were also conducted. In 2005, the Institute of Crop Sciences and Chinese Rice Research Institute of the Chinese Academy of Agricultural Sciences proposed and chaired the construction of the rice gene database. The RiceData database mainly collects genetic rice information (including QTLs), including gene names, functions, locations, and relevant references.

${ }^{1}$ http://www.ricedata.cn/gene/

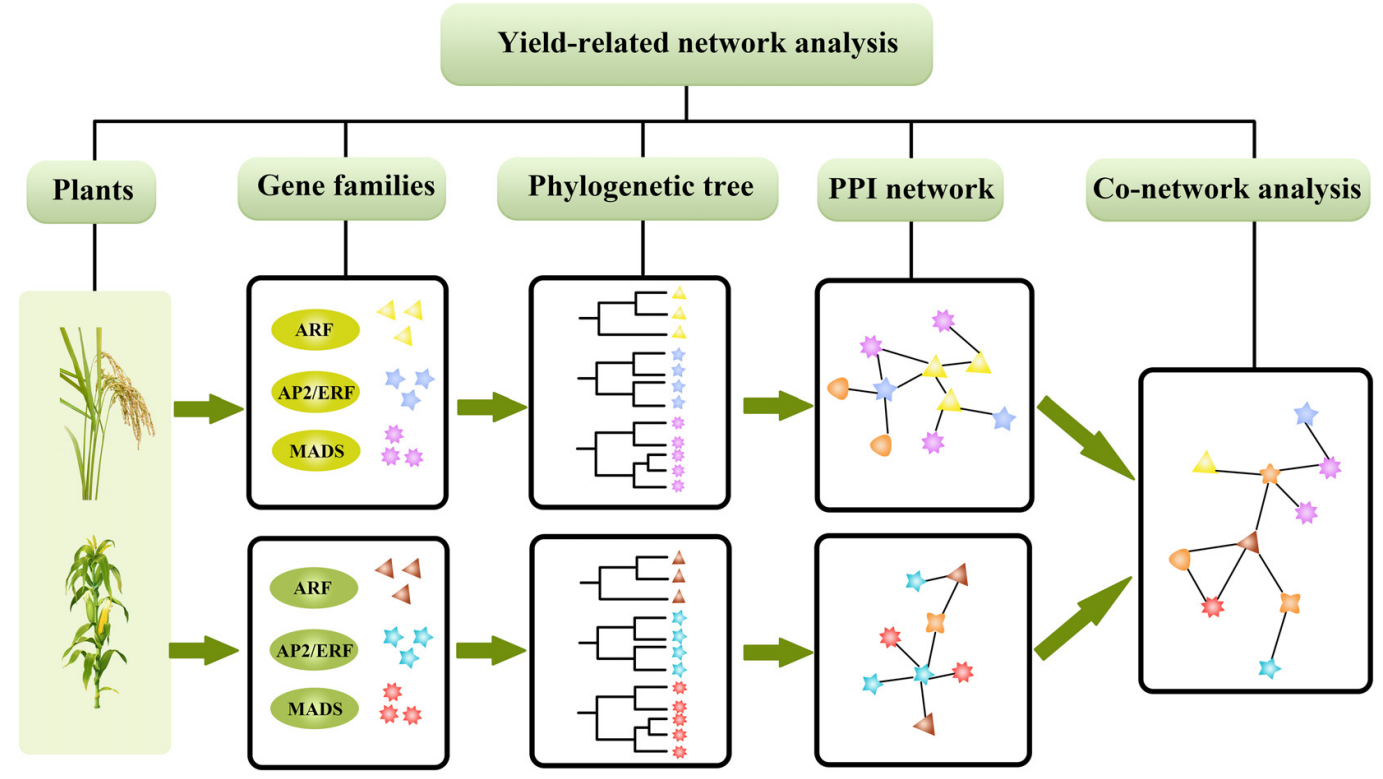

FIGURE 1 | The process of analysis for genetic regulation networks in rice and maize. 
National Center for Biotechnology Information (NCBI)

National Center for Biotechnology Information was established in 1988 to build academic information systems for molecular biology (Sayers et al., 2020). The resources of NCBI comprise six categories: literature, health, genomes, genes, proteins, and chemicals (Sayers et al., 2020). In addition to biological data, NCBI also provides an assortment of analysis and visualization software (Sayers et al., 2020). We obtained sequence data for MADS and AP2 families in maize from NCBI.

\section{Tools}

\section{STRING}

STRING is a web-server ${ }^{2}$ that is widely used to visualize data as interaction networks and to perform gene-set enrichment analysis (Szklarczyk et al., 2019). It collects and integrates known protein-protein interaction (PPI) data from all publicly available sources (Szklarczyk et al., 2019). The source databases include KEGG, Reactome, BioCyc, Gene Ontology and so on (Caspi et al., 2016; Fabregat et al., 2016; Kanehisa et al., 2017; The Gene Ontology Consortium, 2017). STRING interaction predictions are produced by computational prediction efforts, including protein co-expression systems analysis, shared genome shared signal measurement and PubMed abstracts for text mining from all databases, as well as OMIM, and so on.

\section{IQ-TREE}

Phylogenetic analyses have been widely used in molecular systematics. In biology, phylogenetics can be applied in the analysis of the evolutionary relationships among individuals or groups of organisms. IQ-TREE is freely available software for discovering these relationships through phylogenetic inference implementing Maximum likelihood (ML) (Nguyen et al., 2015). The substitution model was calculated with MODELFINDER (integrated in IQ-TREE; best-fit model: JTT + R5 chosen according to the Bayesian information criterion). We also constructed the phylogenetic trees for rice and maize by IQ-TREE.

\section{MAFFT}

Multiple sequence alignments (MSA) is widely used in the alignment of proteins and nucleotide sequences, which are assumed to be inherited from a common ancestor. Detecting co-evolution is a critical step in the prediction of protein-protein interactions (de Juan et al., 2013; Wang et al., 2017). MAFFT is MSA software that offers three alignment strategies, including the progressive method (PartTree, FFT-NS-1, and L-INS-1), iterative refinement methods (FFT-NS-i, L-INS-i, E-INS-i, and G-INS-i) and so on (Katoh and Standley, 2013; Katoh et al., 2019). We aligned protein sequences with their corresponding amino acid domains with MAFFT (Katoh and Standley, 2013; Katoh et al., 2019).

${ }^{2}$ https://string-db.org/

\section{RESULTS}

\section{Identification of AP2 Domain, ARF Domain and MADS Genes in Rice and Maize}

The AP2 domain, ARF domain and MADS gene candidate sequences from rice and maize genomes were derived from the China Rice Data Center (see text footnote 1) and $\mathrm{NCBI}^{3} .300$, 69 and 143 potential sequences were identified as AP2 domaincontaining genes, ARF domain-containing genes and MADS genes, respectively. Detailed information about these genes for rice and maize are provided in Supplementary Tables 1, 2.

\section{Phylogenetic Analysis of AP2 Domain Proteins in Rice and Maize}

We constructed the phylogenetic tree of the AP2 protein sequences in rice and maize to illustrate the phylogenetic relationship. The phylogenetic tree for an AP2 domaincontaining gene family in rice and maize revealed four major clades grouping into 10 subfamilies (Figure 2A). Among the 300 AP2 proteins, 1 belongs to group I, 1 to group II, 3 to group III, and 2 to Group IV. The large groups for AP2 members are VII and VIII. Group VII can be further clustered into four subgroups, besides three subgroups in group VIII. Group I and group II only contain one respective gene each: OsRAV2 and ZmAP2-5. Group III includes three genes of maize, and these groups are the ancient clades. From the dataset, most of the proteins containing the AP2 domain were related to crop yield (Riechmann and Meyerowitz, 1998; Harrop et al., 2019). The yield-related gene OsEATB is in Group VIIIb. Group V includes three close clades, with one clade containing three well-known yield-related genes, OsRSR1, OsSNB, and OsIDS1 (Fu and Xue, 2010; Rashid et al., 2012; Lee et al., 2014; Rao et al., 2014; Ji et al., 2019). The existence of such yield-related proteins was one of the unusual features of the AP2 gene family in flowering plants, such as maize and rice. From the groups or subgroups, the crop yield-associated genes were randomly selected as representatives for further analysis. From previous research, OsEATB can reduce the plant height and panicle length during the maturity stage, promoting the branching potential of both tillers and spikelets (Qi et al., 2011). In rice, the absence of $B B M 1, B B M 2$, and $B B M 3$ would result in embryo arrest and abortion in group V (Khanday et al., 2019). Overexpression of OsAP2-39 can cause a variety of phenotypic changes in transgenic rice, such as the reduction of plant height, tiller, leaf number and 1-2 weeks postponement for heading, ultimately resulting in a decrease in yield due to reduced biomass and grain number (Yaish et al., 2010). OsRSR1 regulates starch synthesis in rice (Fu and Xue, 2010). Compared with the wild type, the grain size is larger and the quality and yield are higher in rsrl (Fu and Xue, 2010). OsIDS1 and OsSNB play important roles in the establishment of inflorescence morphology and floral meristems. There is a significant decrease in branches and spikelets of the inflorescence for the double mutant snb/osids1 plant (Lee and An, 2012). The function of the

\footnotetext{
${ }^{3}$ https://www.ncbi.nlm.nih.gov/
} 

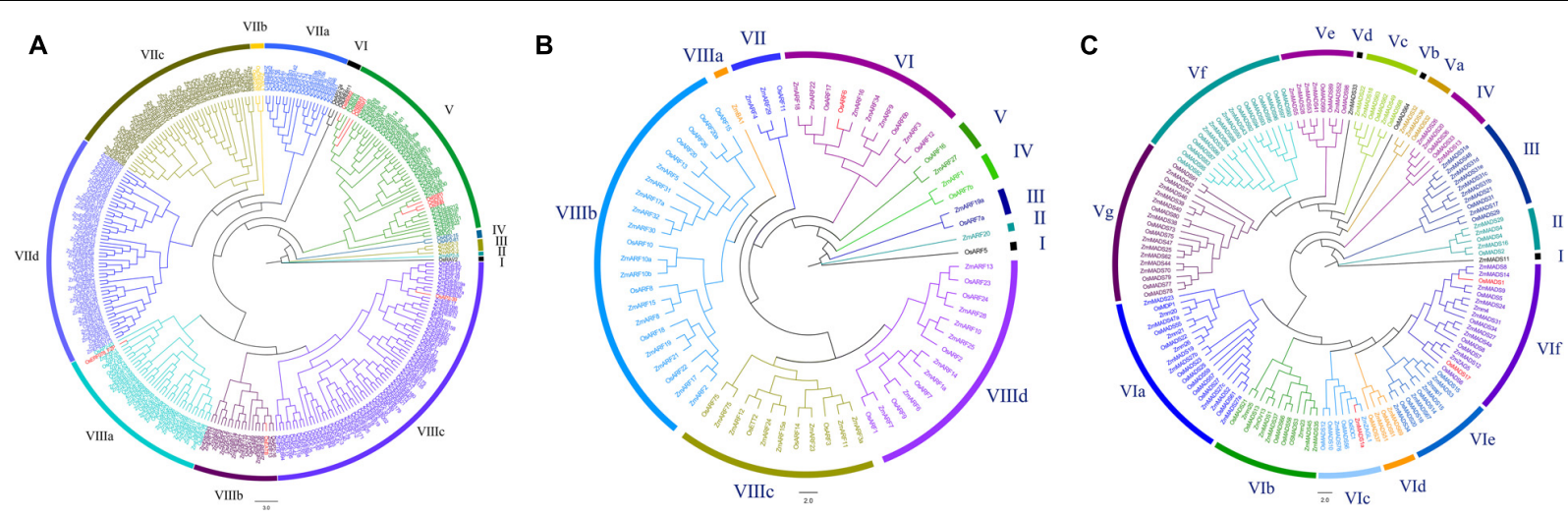

FIGURE 2 | Phylogenetic trees of three gene families in rice and maize (A) Phylogenetic tree of the AP2 domain-containing proteins in rice and maize. Protein sequence alignment using E-INS-i algorithm. AP2 domain-containing proteins grouped into 10 subfamilies. (B) Phylogenetic tree of the ARF proteins in rice and maize. Protein sequence alignment using E-INS-i algorithm. Auxin response factor proteins grouped into 10 subfamilies. (C) Phylogenetic tree of the MADS-box proteins in rice and maize. Protein sequence alignment using E-INS-i algorithm. MADS-box proteins grouped into 17 subfamilies.

OsSNB gene was determined by decreased seed fall, a seed length increase of $7.0 \%$, and a 1000 -seed weight increase of $6.1 \%$ in the ssh1 mutants (Jiang et al., 2019). The SHAT1 gene, which encodes an AP2 transcription factor, is required for seed shattering in rice (Jiang et al., 2019).

\section{Phylogenetic Analysis of ARF Domain Proteins in Rice and Maize}

The full-length amino acid sequences of ARF domain proteins were used for multiple sequence alignment and phylogenetic analyses, respectively. The phylogenetic tree of 69 members of ARF domain-containing genes for rice and maize was constructed (Figure 2B). These ARF domain-containing members can be distinctly divided into eight groups and 11 subfamilies. There is only one gene in groups I and II, OsARF5 and $Z m A R F 20$, respectively, besides two genes confirmed in groups III, IV, and V. Group VI has 10 genes, and group VIII is the largest one of all. The gene OsARF1 in group VIIId was indicated to be essential for growth in vegetative organs and seed development (Attia et al., 2009). Floral organ development is essential to plant yield and seed quality, so overexpression of OsARF19/OsARF7a resulted in high auxin content, dwarfism, shrunken grains and upregulated expression levels of OsMADS29 and OsMADS22, which are two floral organ regulators (Zhang et al., 2015). OsARF2 and OsARF4 are located in the same loci (Wang et al., 2007). During rice grain development, the interaction of OsARF4/OsARF2 and OsSK41 can repress the expression of some auxin responsive genes, and the grain size with respect to osarf4/osarf2 performance is larger (Hu et al., 2018).

\section{Phylogenetic Analysis of MADS Proteins in Rice and Maize}

To understand the evolutionary and phylogenetic relationships of MADS proteins, a phylogenetic tree using E-INS-i algorithm was constructed from rice and maize (Figure 2C). The 143 MADS protein sequences were aligned and classified into six well-supported groups and 17 subfamilies labeled with different colors. According to the phylogenetic tree, there is only one gene in group I, as well as five genes, 10 genes and four genes in groups II, III, and IV, respectively. Group V and VI are larger than others, with seven and six subfamilies, respectively. Based on previous studies, MADS protein functions are related to floral, ovule and seed organ development (Schwarz-Sommer et al., 1990; Becker and Theissen, 2003). For example, OsMADS3, OsMADS13, and DROOPING LEAF play various important roles in floral development (Dreni et al., 2007; Liu et al., 2011). Downregulated expression of OsMADS7 and OsMADS8 resulted in severe phenotype deterioration for plants, including late flowering, abnormal performance of lodicules, stamens and carpels, and a loss of floral determinacy (Cui et al., 2009). OsMADS1 is mainly expressed in flower organs and determines the formation of the lemma and palea (Chung et al., 1994). All of the OsMADS1 transgenic plants exhibited similar phenotypes, including dwarfism, distorted panicles, decreased numbers of branches and spikelets, and elongated sterile lemmas (Jang et al., 2017). The G $\gamma$ subunits interacting with GS3 and DEP1 can activate the expression of OsMADS1 to regulate grain shape (Liu et al., 2018). OsMADS17 expression is regulated by OsMADS1 and involved in hormone signaling and floral identity (Hu et al., 2015).

\section{Prediction and Analysis of Genetic Network for Rice Yield-Related Genes}

Here, the rice yield-related genes belonging to MADS-box, ARF, and AP2 domain-containing gene families were used to construct the genetic network. The AP2 domain-containing protein gene family contains nine proteins: OsEATB, OsRSR1, OsBBM1, OsBBM2, OsBBM3, OsSNB, OsIDS1, OsAP2-39, and OsERF078/FZP. There are three proteins, OsARF1, OsARF19/7a, and OsARF4/OsARF2, belonging to the ARF family. OsMADS1, OsMADS3, OsMADS7, OsMADS8, OsMADS13, and OsMADS17 belong to the MADS-box gene family. The protein sequences are provided in Supplementary File 5. The 


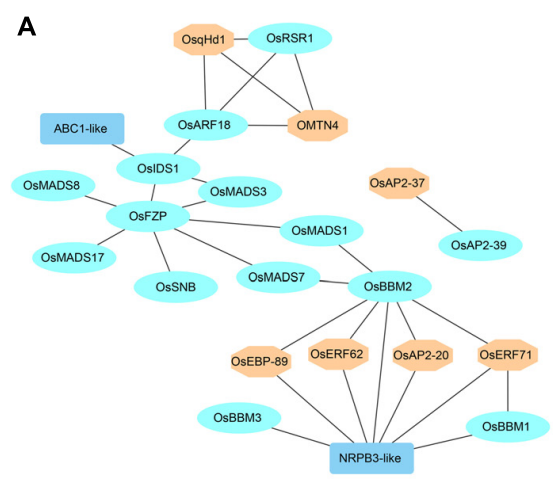

Rice PPI network

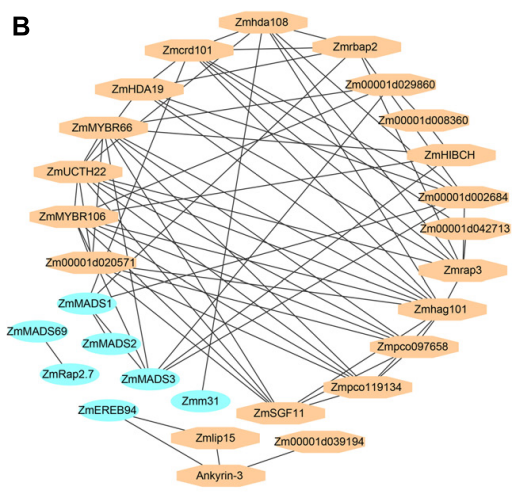

Maize PPI network

FIGURE 3 | The PPI networks of yield-related genes (A) The PPI network of yield-related genes in rice. (B) The PPI network of yield-related genes in maize.

STRING database and Cytoscape_v3.7.2 were used to construct the protein-protein interaction network (PPI) (Szklarczyk et al., 2019) and the PPI network detail information is in Supplementary Table 3. From Figure 3A, there are 14 proteins from the three gene families, as well as seven proteins which have not been cloned. Among the seven proteins, OsqHd1, similar to SBP-domain protein 4, is a minor QTL with the functions of delaying heading and increasing the numbers of spikelets per panicle, grains per panicle and the grain yield per plant (Chen et al., 2014). OsEBP-89, OsERF62, OsERF71, OsAP2-20, and $O s A P 2-37$ indicated that the AP2 domain-containing gene family plays an important role in rice yield. NRPB3-like (Os09g0110400) and OMTN4 (Os06g0675600) belong to the NAC gene family, and overexpression of OMTN4 negatively affected drought tolerance during the rice reproductive stage (Fang et al., 2014). The homologous gene for ABC1-like (Os01g0318700) protein is AtOSA1 (AT4G01660) in Arabidopsis, a member of the ATH subfamily which encodes an ABC transporter (Jasinski et al., 2008).

\section{Prediction and Analysis of Genetic Network for Maize Yield-Related Genes}

Some yield-related genes were randomly selected from the MADS-box, ARF and AP2 domain-containing gene families in maize. The AP2 domain-containing protein gene family contains three proteins, ZmEREB94, ZmEREB156 and ZmRap2.7, as well as ZmMADS1, ZmMADS3, ZmMADS31, Zmm4, ZmMADS1a, and ZmMADS2, which belong to the MADS-box gene family. The protein sequences are provided in Supplementary File 5. The STRING database and Cytoscape_v3.7.2 were used to construct the protein-protein interaction network (PPI) (Szklarczyk et al., 2019) and the PPI network detail information is in Supplementary Table 3. From Figure 3B, there are seven cloned proteins from the three gene families and 21 unknown proteins. Among the 21 proteins, ZmMYBR66 and ZmMYBR106 belong to the MYB gene family, Zm00001d002684 has the function of flower locus D, and Zmhda108 and ZmHDA19 belong to the histone deacetylase family. A large number of uncloned genes were uncharacterized proteins.

\section{Analysis of the Similar Genetic Network for Maize and Rice Yield-Related Genes}

In this research, the yield-related genes were selected from total rice and maize in Supplementary Table 4 and Supplementary File 5. Twenty-eight protein sequences containing 18 rice protein sequences and 10 maize protein sequences were used to construct the co-network using the STRING database. Based on the previous research, there are 13 cloned proteins that interact with the other 18 new proteins (Figure 4).

OsERF71, OsAP2-20, OsAP2-37, NRPB3-like (Os09g0110400), and OMTN4 (Os06g0675600) suggested that the AP2 domaincontaining and NAC gene family serve important roles in rice yield. OsqHd1 also takes part in increasing the number of grains per panicle and grain yield (Chen et al., 2014). Overexpression of OsMADS56 also resulted in delayed flowering in the situation

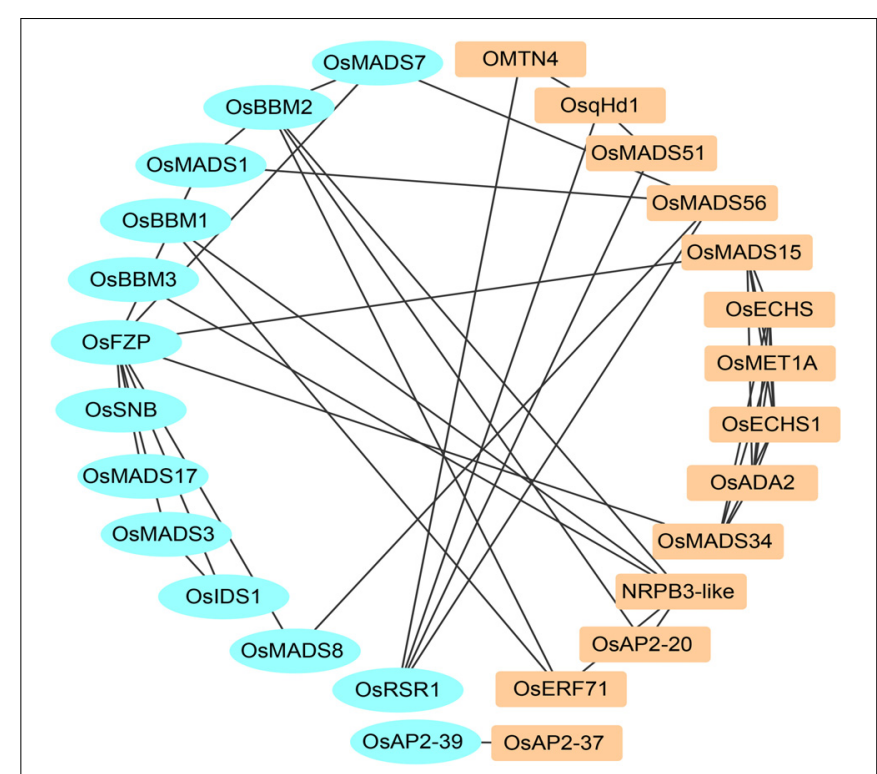

FIGURE 4 | The PPI network of rice and maize yield-related genes. 
of long days (Ryu et al., 2009). There is an aberrant phenotype of the mutant osmads34- $t$ compared with the wild plants, such as more primary branch numbers, abnormal panicles, and the length of sterile lemmas: therefore, OsMADS34 is involved in rice yield and grain size (Kobayashi et al., 2009, 2012; Gao et al., 2010; Yu et al., 2016). Loss of OsMADS51 exerted little effect on flowering in long days, and OsMADS51 can transmit an SD promotion signal from OsGI to Ehd1 as a novel flowering promoter (Kim et al., 2008). The axillary buds exhibited accelerated development and frequently grew into effective tillers upon overexpression of OsMADS15. The panicles were large in the OsMADS15 transgenic rice (Lu et al., 2012). OsMET1A is a DNA methyltransferase which is primarily responsible for maintaining CpG methylation (Yamauchi et al., 2008). OsECHS, OsECHS1 and OsADA2 are three uncharacterized proteins. These results suggested that most proteins in the predicted network are yield-related genes.

\section{DISCUSSION}

A large diversity of agronomic traits are important determinants of yield in rice and maize, such as grain size, shape weight, spikelets and tillers per plant, among others. Brassinolide (BR) and auxin, as the most important plant hormones, serve important roles in grain development and regulate factors such as grain size, shape, and weight.

Maize and rice share a common ancestor. Similar traits were usually controlled by QTLs in syntenic regions among species. Many genes that may affect seed shape and weight have been mapped and cloned in rice, such as GS3, GW2, and GS5. GS3 and GW2 were isolated for maize orthologs genes of rice, and found that maize genes also controlled similar traits. AP2 domain, ARF domain, and MADS genes families are involved in the determinants of yield. Previous studies have suggested that AP2 functions as a nuclear transcription factor in plant cells. Auxin and BR serve important roles in the development of seeds and genes (Zuo and Li, 2014). The ARF family members function as transcriptional activators and repressors in plants (Guilfoyle, 2015). In addition, ARF18 impacts the expression of the downstream auxin-responsive genes and affects silique length and seed weight (Liu et al., 2015b). However, previous research indicates that rice grain weight, grain size, grain hull, endosperm development and activity in maternal tissues are

\section{REFERENCES}

Alvarez-Buylla, E. R., Garcia-Ponce, B., Sanchez, M. P., Espinosa-Soto, C., GarciaGomez, M. L., Pineyro-Nelson, A., et al. (2019). MADS-box genes underground becoming mainstream: plant root developmental mechanisms. New. Phytol. 223, 1143-1158. doi: 10.1111/nph.15793

Attia, K. A., Abdelkhalik, A. F., Ammar, M. H., Wei, C., Yang, J., Lightfoot, D. A., et al. (2009). Antisense phenotypes reveal a functional expression of OsARF1, an auxin response factor, in transgenic rice. Curr. Issues Mol. Biol. 11(Suppl. 1), i29-i34.

Becker, A., and Theissen, G. (2003). The major clades of MADS-box genes and their role in the development and evolution of flowering negatively regulated by OsARF4 ( $\mathrm{Hu}$ et al., 2018). In addition, WRINKLED1 (WRI1), as the AP2/EREBP transcription factor in Arabidopsis, also serves the function of seed storage metabolism (Maeo et al., 2009). In rice, OsERF2 mediated gene expression in the metabolism of sucrose and plant hormone signaling pathways affecting the accumulation of sucrose and UDPG (Maeo et al., 2009). The MADS family is a group of crucial regulatory factors that control the development of floral organs: for example, the OsMADS1 gene can induce flowering (Chung et al., 1994).

In our study, there were several genes predicted to participate in the yield-related network, and these genes were uncharacterized genes belonging to diverse gene families. Some genes were known as yield-related genes, OsqHd1, an SBPdomain protein, serves the function of delaying heading and increasing grain yield. NRPB3-like was a predicted gene of the NAC gene family, and ABC1-like was an aarF domain-containing protein kinase. These genes are novel, so further studies on the functions of these unknown genes are necessary.

\section{DATA AVAILABILITY STATEMENT}

The original contributions presented in the study are included in the article/Supplementary Material, further inquiries can be directed to the corresponding authors.

\section{AUTHOR CONTRIBUTIONS}

QZ designed the research. ZC and ZS performed the research and wrote the manuscript. ZC, ZS, and DZ analyzed the data. All authors read and approved the manuscript.

\section{FUNDING}

This work was supported by the National Natural Science Foundation of China (No. 91935302).

\section{SUPPLEMENTARY MATERIAL}

The Supplementary Material for this article can be found online at: https://www.frontiersin.org/articles/10.3389/fcell.2020. 621464/full\#supplementary-material

plants. Mol. Phylogenet. Evol. 29, 464-489. doi: 10.1016/s1055-7903(03) 00207-0

Bommert, P., Satoh-Nagasawa, N., and Jackson, D. (2013). Quantitative variation in maize kernel row number is controlled by the FASCIATED EAR2 locus. Nat. Genet. 45, 334-337. doi: 10.1038/ng.2534

Caspi, R., Billington, R., Ferrer, L., Foerster, H., Fulcher, C. A., Keseler, I. M., et al. (2016). The MetaCyc database of metabolic pathways and enzymes and the BioCyc collection of pathway/genome databases. Nucleic Acids Res. 44, D471-D480. doi: 10.1093/nar/gkv 1164

Chen, J.-Y., Guo, L., Ma, H., Chen, Y.-Y., Zhang, H.-W., Ying, J., et al. (2014). Fine mapping of qHd1, a minor heading date QTL with pleiotropism for yield 
traits in rice (Oryza sativa L.). Theor. Appl. Genet. 127, 2515-2524. doi: 10.1007/ s00122-014-2395-7

Chu, Y., Xu, N., Wu, Q., Yu, B., Li, X., Chen, R., et al. (2019). Rice transcription factor OsMADS57 regulates plant height by modulating gibberellin catabolism. Rice 2:38. doi: 10.1186/s12284-019-0298-6

Chung, Y. Y., Kim, R., Finkel, D., Yanofsky, M., and An, G. (1994). Early flowering and reduced apical dominance result from ectopic expression of a rice MADS box gene. Plant mol. Biol. 26, 657-665. doi: 10.1007/BF00013751

Cui, R., Han, J., Zhao, S., Su, K., Wu, F., Du, X., et al. (2009). Functional conservation and diversification of class $\mathrm{E}$ floral homeotic genes in rice (Oryza sativa). Plant J. 61, 767-781. doi: 10.1111/j.1365-313X.2009.04101.x

de Juan, D., Pazos, F., and Valencia, A. (2013). Emerging methods in protein co-evolution. Nat. Rev. Genet. 14, 249-261. doi: 10.1038/nrg3414

Dreni, L., Jacchia, S., Fornara, F., Fornari, M., Ouwerkerk, P., An, G., et al. (2007). The D-lineage MADS-box gene OsMADS13 controls ovule identity in rice. Plant J. 52, 690-699. doi: 10.1111/j.1365-313X.2007.03272.x

Fabregat, A., Sidiropoulos, K., Garapati, P., Gillespie, M., Hausmann, K., Haw, R., et al. (2016). The reactome pathway knowledgebase. Nucleic Acids Res. 44, D481-D487. doi: 10.1093/nar/gkv1351

Fang, Y., Xie, K., and Xiong, L. (2014). Conserved miR164-targeted NAC genes negatively regulate drought resistance in rice. J. Exp. Bot. 65, 2119-2135. doi: 10.1093/jxb/eru072

Fu, F. F., and Xue, H. W. (2010). Coexpression analysis identifies rice starch regulator1, a rice AP2/EREBP family transcription factor, as a novel rice starch biosynthesis regulator. Plant Physiol. 154, 927-938. doi: 10.1104/pp.110.159517

Gao, X., Liang, W., Yin, C., Ji, S., Wang, H., Su, X., et al. (2010). The SEPALLATA-like gene OsMADS34 is required for rice inflorescence and spikelet development. Plant Physiol. 153, 728-740. doi: 10.1104/pp.110.156711

Gramzow, L., Ritz, M. S., and Theissen, G. (2010). On the origin of MADS-domain transcription factors. Trends Genet. 26, 149-153. doi: 10.1016/j.tig.2010.01.004

Guilfoyle, T. (2015). The PB1 domain in auxin response factor and Aux/IAA proteins: a versatile protein interaction module in the auxin response. Plant Cell 27, 33-43. doi: 10.1105/tpc.114.132753

Harrop, T. W. R., Mantegazza, O., Luong, A. M., Bethune, K., Lorieux, M., Jouannic, S., et al. (2019). A set of AP2-like genes is associated with inflorescence branching and architecture in domesticated rice. J. Exp. Bot. 70, 5617-5629. doi: 10.1093/jxb/erz340

Hu, Y., Liang, W., Yin, C., Yang, X., Ping, B., Li, A., et al. (2015). Interactions of OsMADS1 with floral homeotic genes in rice flower development. Mol. Plant 8, 1366-1384. doi: 10.1016/j.molp.2015.04.009

Hu, Z., Lu, S.-J., Wang, M.-J., He, H., Sun, L., Wang, H., et al. (2018). A novel QTL qTGW3 Encodes the GSK3/SHAGGY-like kinase OsGSK5/OsSK41 that interacts with OsARF4 to negatively regulate grain size and weight in rice. Mol. Plant 11, 736-749. doi: 10.1016/j.molp.2018.03.005

Jang, S., Li, H.-Y., and Kuo, M.-L. (2017). Ectopic expression of Arabidopsis FD and FD paralogue in rice results in dwarfism with size reduction of spikelets. Sci. Rep. 7:44477. doi: 10.1038/srep44477

Jasinski, M., Sudre, D., Schansker, G., Schellenberg, M., Constant, S., Martinoia, E., et al. (2008). AtOSA1, a member of the abcl-like family, as a new factor in cadmium and oxidative stress response. Plant Physiol. 147, 719-731. doi: 10.1104/pp.107.110247

Ji, H., Han, C. D., Lee, G. S., Jung, K. H., Kang, D. Y., Oh, J., et al. (2019). Mutations in the microRNA172 binding site of supernumerary bract (SNB) suppress internode elongation in rice. Rice 12:62. doi: 10.1186/s12284-019-0324-8

Jiang, L., Ma, X., Zhao, S., Tang, Y., Liu, F., Gu, P., et al. (2019). The Apetala2-like transcription factor supernumerary bract controls rice seed shattering and seed size. Plant Cell 31, 17-36. doi: 10.1105/tpc.18.00304

Kanehisa, M., Furumichi, M., Tanabe, M., Sato, Y., and Morishima, K. (2017). KEGG: new perspectives on genomes, pathways, diseases and drugs. Nucleic Acids Res. 45, D353-D361. doi: 10.1093/nar/gkw1092

Katoh, K., Rozewicki, J., and Yamada, K. D. (2019). MAFFT online service: multiple sequence alignment, interactive sequence choice and visualization. Brief. Bioinform. 20, 1160-1166. doi: 10.1093/bib/bbx108

Katoh, K., and Standley, D. M. (2013). MAFFT multiple sequence alignment software version 7: improvements in performance and usability. Mol. Biol. Evol. 30, 772-780. doi: 10.1093/molbev/mst010
Khanday, I., Skinner, D., Yang, B., Mercier, R., and Sundaresan, V. (2019). A maleexpressed rice embryogenic trigger redirected for asexual propagation through seeds. Nature 565, 91-95. doi: 10.1038/s41586-018-0785-8

Kim, S., Lee, S., Kim, H., Nam, H., and An, G. (2008). OsMADS51 is a short-day flowering promoter that functions upstream of Ehd1, OsMADS14, and Hd3a. Plant Physiol. 145, 1484-1494. doi: 10.1104/pp.107.103291

Kobayashi, K., Maekawa, M., Miyao, A., Hirochika, H., and Kyozuka, J. (2009). Panicle Phytomer2 (PAP2), encoding a SEPALLATA subfamily MADS-box protein, positively controls spikelet meristem identity in rice. Plant Cell Physiol. 51, 47-57. doi: 10.1093/pcp/pcp166

Kobayashi, K., Yasuno, N., Sato, Y., Yoda, M., Yamazaki, R., Kimizu, M., et al. (2012). Inflorescence meristem identity in rice is specified by overlapping functions of three AP1/FUL-like MADS box genes and PAP2, a SEPALLATA MADS box gene. Plant Cell 24, 1848-1859. doi: 10.1105/tpc.112.097105

Lee, D.-Y., and An, G. (2012). Two AP2 family genes, supernumerary bract (SNB) and Osindeterminate spikelet 1 (OsIDS1), synergistically control inflorescence architecture and floral meristem establishment in rice. Plant J. 69, 445-461. doi: 10.1111/j.1365-313x.2011.04804.x

Lee, Y. S., Lee, D. Y., Cho, L. H., and An, G. (2014). Rice miR172 induces flowering by suppressing OsIDS1 and SNB, two AP2 genes that negatively regulate expression of Ehd1 and florigens. Rice 7:31. doi: 10.1186/s12284-014-0031-4

Li, H., Xiao, Q., Zhang, C., Du, J., Li, X., Huang, H., et al. (2017). Identification and characterization of transcription factor ZmEREB94 involved in starch synthesis in maize. J. Plant Physiol. 216, 11-16. doi: 10.1016/j.jplph.2017.04.016

Li, J., Chen, F., Li, Y., Li, P., Wang, Y., Mi, G., et al. (2019). ZmRAP2.7, an AP2 transcription factor, is involved in maize brace roots development. Front. Plant Sci. 10:820. doi: 10.3389/fpls.2019.00820

Li, S. B., Xie, Z. Z., Hu, C. G., and Zhang, J. Z. (2016). A review of auxin response factors (ARFs) in plants. Front. Plant Sci. 7:47. doi: 10.3389/fpls.2016.00047

Liang, Y., Liu, Q., Wang, X., Huang, C., Xu, G., Hey, S., et al. (2019). ZmMADS69 functions as a flowering activator through the ZmRap2.7-ZCN8 regulatory module and contributes to maize flowering time adaptation. New Phytol. 221, 2335-2347. doi: 10.1111/nph.15512

Liu, J., Deng, M., Guo, H., Luo, J., Xu, Y., Dong, X., et al. (2015a). Maize orthologs of rice GS5 and their trans- regulator are associated with kernel development. J. Integr. Plant Biol. 57, 943-953. doi: 10.1111/jipb.12421

Liu, J., Hua, W., Hu, Z., Zhang, L., Li, R., Deng, L., et al. (2015b). Natural variation in ARF18 gene simultaneously affects seed weight and silique length in polyploid rapeseed. Proc. Natl. Acad. Sci. U.S.A. 112, E5123-E5132. doi: 10.1073/pnas.1502160112

Liu, L., Du, Y., Shen, X., Li, M., Sun, W., Huang, J., et al. (2015c). KRN4 controls quantitative variation in maize kernel row number. PLoS Genet. 11:1005670. doi: 10.1371/journal.pgen.1005670

Liu, J., Huang, J., Guo, H., Lan, L., Wang, H., Xu, Y., et al. (2017). The conserved and unique genetic architecture of kernel size and weight in maize and rice. Plant Physiol. 175, 774-785. doi: 10.1104/pp.17.00708

Liu, Q., Han, R., Wu, K., Zhang, J., Ye, Y., Shuansuo, W., et al. (2018). G-protein $\beta \gamma$ subunits determine grain size through interaction with MADS-domain transcription factors in rice. Nat. Commun. 9:852. doi: 10.1038/s41467-01803047-9

Liu, R., Liang, W., Yin, C., Zhu, L., and Zhang, D. (2011). Genetic interaction of OsMADS3, DROOPING LEAF, and OsMADS13 in specifying rice floral organ identities and meristem determinacy. Plant Physiol. 156, 263-274. doi: 10.1104/pp.111.172080

Lu, S.-J., Wei, H., Wang, Y., Wang, H.-M., Yang, R.-F., Zhang, X.-B., et al. (2012). Overexpression of a transcription factor OsMADS15 modifies plant architecture and flowering time in rice (Oryza sativa L.). Plant Mol. Biol. Report. 30, 1461-1469. doi: 10.1007/s11105-012-0468-9

Maeo, K., Tokuda, T., Ayame, A., Mitsui, N., Kawai, T., Tsukagoshi, H., et al. (2009). An AP2-type transcription factor, WRINKLED1, of Arabidopsis thaliana binds to the AW-box sequence conserved among proximal upstream regions of genes involved in fatty acid synthesis. Plant J. 60, 476-487. doi: 10.1111/j.1365-313X. 2009.03967.x

Nguyen, L. T., Schmidt, H. A., von Haeseler, A., and Minh, B. Q. (2015). IQ-TREE: a fast and effective stochastic algorithm for estimating maximum-likelihood phylogenies. Mol. Biol. Evol. 32, 268-274. doi: 10.1093/molbev/msu300 
Paul, P., Dhatt, B. K., Miller, M., Folsom, J. J., Wang, Z., Krassovskaya, I., et al. (2020). MADS78 and MADS79 are essential regulators of early seed development in rice. Plant Physiol. 182, 933-948. doi: 10.1104/pp.19.00917

Qi, W., Sun, F., Wang, Q., Chen, M., Huang, Y., Feng, Y.-Q., et al. (2011). Rice ethylene-response AP2/ERF factor OsEATB restricts internode elongation by down-regulating a gibberellin biosynthetic gene. Plant Physiol. 157, 216-228. doi: $10.1104 /$ pp.111.179945

Rao, Y., Li, Y., and Qian, Q. (2014). Recent progress on molecular breeding of rice in China. Plant Cell Rep. 33, 551-564. doi: 10.1007/s00299-013-1551-x

Rashid, M., Guangyuan, H., Guangxiao, Y., Hussain, J., and Xu, Y. (2012). AP2/ERF transcription factor in rice: genome-wide canvas and syntenic relationships between monocots and eudicots. Evol. Bioinform. Online 8, 321355. doi: 10.4137/EBO.S9369

Ray, D. K., Mueller, N. D., West, P. C., and Foley, J. A. (2013). Yield trends are insufficient to double global crop production by 2050. PLoS One 8:e66428. doi: 10.1371 /journal.pone.0066428

Riechmann, J. L., and Meyerowitz, E. M. (1998). The AP2/EREBP family of plant transcription factors. Biol. Chem. 379, 633-646. doi: 10.1515/bchm.1998.379.6. 633

Ryu, C.-H., Lee, S., Cho, L.-H., Kim, S., Lee, Y.-S., Choi, S. C., et al. (2009). OsMADS50 and OsMADS56 function antagonistically in regulating long day (LD)-dependent flowering in rice. Plant Cell Environ. 32, 1412-1427. doi: 10. 1111/j.1365-3040.2009.02008.x

Sayers, E. W., Beck, J., Brister, J. R., Bolton, E. E., Canese, K., Comeau, D. C., et al. (2020). Database resources of the national center for biotechnology information. Nucleic Acids Res. 48, D9-D16. doi: 10.1093/nar/gkz899

Schwarz-Sommer, Z., Huijser, P., Nacken, W., Saedler, H., and Sommer, H. (1990). Genetic control of flower development by homeotic genes in antirrhinum majus. Science 250, 931-936. doi: 10.1126/science.250.4983.931

Szklarczyk, D., Gable, A. L., Lyon, D., Junge, A., Wyder, S., Huerta-Cepas, J., et al. (2019). STRING v11: protein-protein association networks with increased coverage, supporting functional discovery in genome-wide experimental datasets. Nucleic Acids Res. 47, D607-D613. doi: 10.1093/nar/gky1131

The Gene Ontology Consortium. (2017). Expansion of the gene ontology knowledgebase and resources. Nucleic Acids Res. 45, D331-D338. doi: 10.1093/ nar/gkw1108

Wang, D., Pei, K., Fu, Y., Sun, Z., Li, S., Liu, H., et al. (2007). Genome-wide analysis of the auxin response factors (ARF) gene family in rice (Oryza sativa). Gene 394, 13-24. doi: 10.1016/j.gene.2007.01.006

Wang, J., Lin, Z., Zhang, X., Liu, H., Zhou, L., Zhong, S., et al. (2019). krn1, a major quantitative trait locus for kernel row number in maize. New Phytol. 223, 1634-1646. doi: 10.1111/nph.15890

Wang, S., Sun, S., Li, Z., Zhang, R., and Xu, J. (2017). Accurate de novo prediction of protein contact map by ultra-deep learning model. PLoS Comput. Biol. 13:e1005324. doi: 10.1371/journal.pcbi.1005324

Wang, Y., and Li, J. (2011). Branching in rice. Curr. Opin. Plant Biol. 14, 94-99. doi: $10.1016 /$ j.pbi.2010.11.002
Wu, J., Lawit, S. J., Weers, B., Sun, J., Mongar, N., Van Hemert, J., et al. (2019). Overexpression of zmm28 increases maize grain yield in the field. Proc. Natl. Acad. Sci. U.S.A. 116, 23850-23858. doi: 10.1073/pnas.1902593116

Xie, Y., Yu, X., Jiang, S., Xiao, K., Wang, Y., Li, L., et al. (2020). OsGL6, a conserved AP2 domain protein, promotes leaf trichome initiation in rice. Biochem. Biophys. Res. Commun. 522, 448-455. doi: 10.1016/j.bbrc.2019.11.125

Yaish, M., El-kereamy, A., Zhu, T., Beatty, P., Good, A., Bi, Y.-M., et al. (2010). The APETALA-2-like transcription factor OsAP2-39 controls key interactions between abscisic acid and gibberellin in rice. PLoS Genet. 6:e1001098. doi: 10.1371/journal.pgen.1001098

Yamauchi, T., Moritoh, S., Johzuka-Hisatomi, Y., Ono, A., Terada, R., Nakamura, I., et al. (2008). Alternative splicing of the rice OsMET1 genes encoding maintenance DNA methyltransferase. J. Plant Physiol. 165, 1774-1782. doi: 10.1016/j.jplph.2007.12.003

Yan, J., and Tan, B. C. (2019). Maize biology: from functional genomics to breeding application. J. Integr. Plant Biol. 61, 654-657. doi: 10.1111/jipb. 12819

Yang, J., Yeh, C.-T. E., Ramamurthy, R. K., Qi, X., Fernando, R. L., Dekkers, J. C. M., et al. (2018). Empirical comparisons of different statistical models to identify and validate kernel row number-associated variants from structured multiparent mapping populations of maize. G3 8, 3567-3575. doi: 10.1534/g3.118. 200636

Yu, Z., Yu, H., Liu, J., Wang, W., Sun, J., Gao, Q., et al. (2016). Loss of function of OsMADS34 leads to large sterile lemma and low grain yield in rice (Oryza sativa L.). Mol. Breed. 36:147. doi: 10.1007/s11032-016-0578-4

Zhang, P., Allen, W., Nagasawa, N., Ching, A., Heppard, E., Hui, L., et al. (2012). A transposable element insertion within ZmGE2 gene is associated with increase in embryo to endosperm ratio in maize. Theor. Appl. Genet. 125, 1463-1471. doi: 10.1007/s00122-012-1926-3

Zhang, S., Wu, T., Liu, S., Liu, X., Jiang, L., and Wan, J. (2015). Disruption of OsARF19 is critical for floral organ development and plant architecture in rice (Oryza sativa L.). Plant Mol. Biol. Report. 34, 748-760. doi: 10.1007/s11105015-0962-y

Zuo, J., and Li, J. (2014). Molecular genetic dissection of quantitative trait loci regulating rice grain size. Annu. Rev. Genet. 48, 99-118. doi: 10.1146/annurevgenet-120213-092138

Conflict of Interest: The authors declare that the research was conducted in the absence of any commercial or financial relationships that could be construed as a potential conflict of interest.

Copyright (c) 2020 Chen, Shen, Xu, Zhao and Zou. This is an open-access article distributed under the terms of the Creative Commons Attribution License (CC BY). The use, distribution or reproduction in other forums is permitted, provided the original author(s) and the copyright owner(s) are credited and that the original publication in this journal is cited, in accordance with accepted academic practice. No use, distribution or reproduction is permitted which does not comply with these terms. 